\title{
Auger Highlights
}

\author{
Antonella Castellina ${ }^{1}$ for the Pierre Auger Collaboration ${ }^{2}$ \\ ${ }^{1}$ Istituto Nazionale di Astrofisica - OATo and INFN Torino \\ ${ }^{2}$ Observatorio Pierre Auger, Av. San Martín Norte 304, 5613 Malargüe, Argentina \\ (Full author list : http://www.auger.org/archive/authors_2012_12.html)
}

Corresponding author: castellina@to.infn.it

\begin{abstract}
The Pierre Auger Observatory has been designed to investigate the origin and nature of the ultra high energy cosmic rays using a hybrid detection technique. A review of selected results is presented, with the emphasis given to the measurement of energy spectrum, mass composition and arrival directions.
\end{abstract}

Keywords: ultra high energy cosmic rays - sources - energy spectrum - primary composition.

\section{Introduction}

Understanding the sources, nature and propagation properties of the cosmic rays at ultra high energies $\left(E>10^{18} \mathrm{eV}\right)$ is one of the key questions in astroparticle physics. From the experimental point of view, their study can be performed indirectly, by exploiting the Extensive Air Showers they produce by interacting with the nuclei in the Earth atmosphere.

Among the different features characterizing the spectral shape, the region between $\simeq 10^{18}-10^{19} \mathrm{eV}$ is thought to host the transition from galactic to extragalactic cosmic rays. Different models explain it as due to $e^{+} / e^{-}$pair production of protons with the photons of the cosmic microwave background (CMB) [1], or more traditionally to the intersection of a steep galactic component and the onset of a flatter extragalactic one [2].

At even higher energies, above $\simeq 410^{19} \mathrm{eV}$, a cut-off in the cosmic ray flux is expected, due to photo-pion production of extragalactic protons in the CMB (the "GZK cut-off" [3]) , although the same feature could arise when reaching the limits in the maximum energy of the sources.

However, the all particle spectrum cannot provide a discrimination among the different hypotheses, and the determination of the primary composition is mandatory to reach any reliable conclusion. The analysis of the arrival directions and their anisotropy can give further insight into the sources and provide information about the magnetic fields which the ultra high energy (UHE) cosmic rays experience during their travel to Earth.

The Pierre Auger Observatory has been specifically designed to investigate the origin and the nature of ultra high energy cosmic rays. It is located in Malargüe, Ar- gentina, and consists of a surface array (SD) of 1660 water Cherenkov stations on an area of $\simeq 3000 \mathrm{~km}^{2}$, overlooked by 27 air fluorescence telescopes (FD) grouped in four sites [4]. Thanks to the possibility of combining the information from the surface array, measuring the lateral distributions of secondary particles at the ground, and the fluorescence telescopes, observing the longitudinal profile, the reconstruction capabilities are enhanced with respect to the individual detector components.

\section{The Energy Spectrum}

The energy spectrum above $2.510^{18} \mathrm{eV}$ has been determined using the data from the SD [5], considering only events up to $60^{\circ}$. The exposure is obtained by integrating the number of active stations over time; the overall acceptance uncertainty is $\simeq 3 \%$ [6]. The energy calibration is derived directly from data, using a subset of high quality hybrid events, i.e. events reconstructed by both the FD and the SD [7]. Despite the low duty cycle of the FD, the energy spectrum could be extended to $10^{18} \mathrm{eV}$ using hybrid events, thanks to the good energy resolution and low threshold, thus investigating the transition region in detail [8]. The total systematic uncertainty in the energy scale is about $22 \%$, the main contribution coming from the uncertainty in the fluorescence yield (14\%) and in the reconstruction of the longitudinal profile (10\%).

The SD and hybrid spectra can be combined using a maximum likelihood method, since both have the same systematic uncertainties in the energy scale. The normalization uncertainties are on the contrary independent and have been used as additional constraints in the 
procedure. The resulting spectrum is shown in Fig.1; a fit with three power laws is shown by the dashed lines, while the solid line indicates the result of a fit with two power laws and a smooth function. The ankle feature is present at an energy of $10^{18.62} \mathrm{eV}$; the cutoff is clearly seen with a significance of $20 \sigma$. Different astrophysical models can be compared to our data; however, the energy spectrum can be described by both a heavy or proton composition at the highest energies and the information must be complemented by independent measurements of the primary composition.

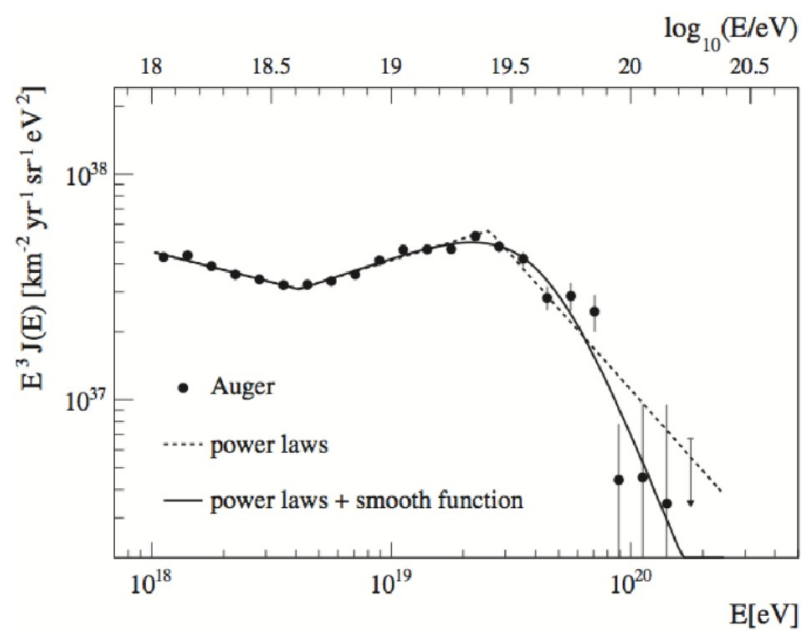

Figure 1: The combined Auger energy spectrum. Only statistical uncertainties are shown. The systematic uncertainty on the energy scale is $22 \%$.

A comparison of the Auger results with data from HiRes, Telescope Array and Yakutsk has been recently performed [9]. The various fluxes can be rescaled assuming that any difference among them be due solely to energy scale and not to aperture calculations or energy resolution. The differences found are entirely consistent with the systematic energy uncertainties quoted by the experiments.

\section{The Nature of the Primaries}

The most direct information about composition can be obtained by measuring the longitudinal development of showers in hybrid events, thus determining the mean depth of maximum development, $X_{\max }$, and its fluctuation, $R M S\left(X_{\max }\right)$. For each event, $X_{\max }$ depends on the depth of first interaction of the primary in the atmosphere and on the subsequent development of the shower; for this reason, the interpretation of the results in terms of composition is complicated by the uncertainties in the hadronic interaction models used in simulations.

About six years of hybrid data have been analyzed applying FD quality cuts and ensuring that no bias with respect to the cosmic ray composition is introduced in the data sample [10]. Having been corrected for the detector resolution, the $X_{\max }$ and its fluctuations are detector independent and can be directly compared to the predictions of different models, as shown in Fig.2. Both observables show a change for $E>510^{18} \mathrm{eV}$ towards an increasingly heavy composition in comparison to the model predictions. The average resolution of $X_{\max } \simeq 20 \mathrm{~g} / \mathrm{cm}^{2}$ in the considered energy range.
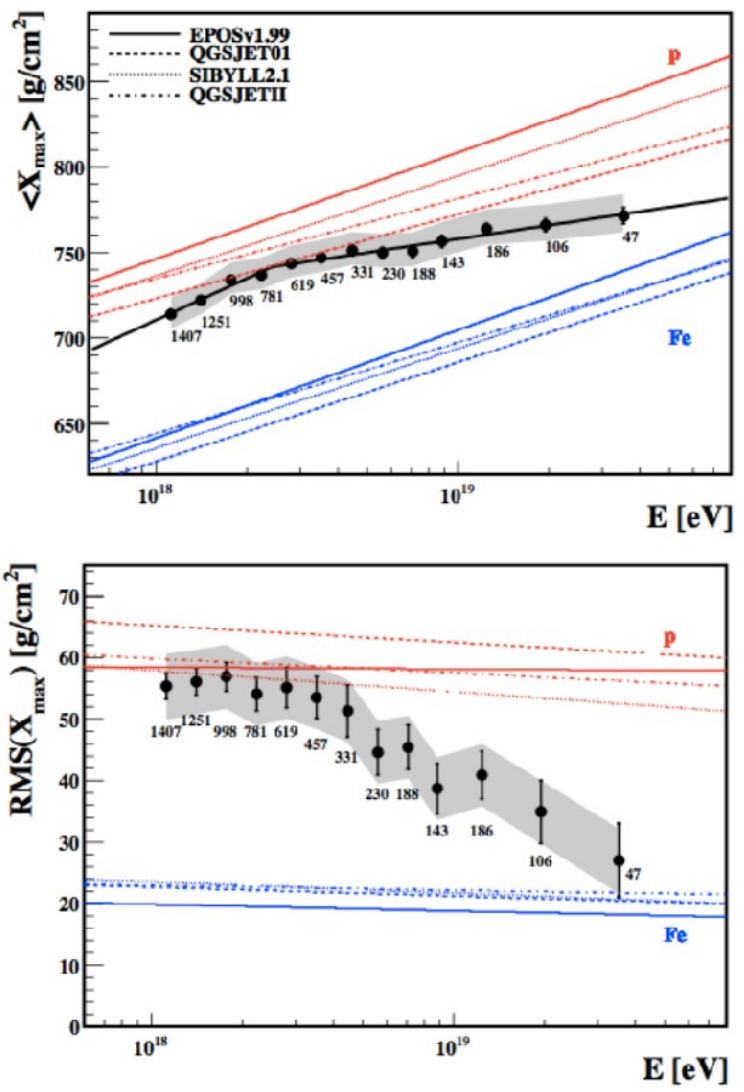

Figure 2: $\left\langle X_{\max }>\right.$ and $R M S\left(X_{\max }\right)$ as a function of energy, compared with the predictions of air shower simulations using different hadronic interaction models.

In general, different values for $\operatorname{RMS}\left(X_{\max }\right)$ are allowed for different combinations of elements [11]. The fluctuations predicted by the considered hadronic interaction models and shown in Fig. 2 are evaluated only for pure compositions .

A different conclusion, leading to a light composition up to the highest energies, has been drawn from the data of the HiRes and Telescope Array. However, a direct comparison of their results with the Auger ones is not possible, as the detector biases are included in their simulation. Furthermore, their dataset is smaller that that of Auger. A lenghty discussion about this comparison can be found in [12] (and refs. therein).

Starting from an extension of the Heitler model of ex- 
tensive air showers [13], a method for interpreting the results of $X_{\max }$ and $R M S\left(X_{\max }\right)$ in terms of mass composition has been developed. As discussed in [14], $X_{\max }$ is only function of the mean logarithmic mass $\langle\ln A\rangle$, and as such it carries information on the average composition. On the contrary, both the shower-to-shower fluctuations and the dispersion in the mass distribution contribute to $R M S\left(X_{\max }\right)$. This information can be used to extract the $\langle\ln A\rangle$ and its variance from the observables and to build the plots shown in Fig.3, where the size of the data points increases with increasing energy.

The energy evolution of the composition is common to all models; reduced systematic uncertainties will allow in the future to test or even exclude some of them.

Different consequences from the astrophysical point of view can be derived from this comparison.

Extragalactic sources of protons seem to be disfavored by our composition result, within the uncertainties on the hadronic interaction models used to interpret the data. In a propagation scenario, nuclei from nearby sources could produce small mass dispersion at Earth, as propagation would not be able to degrade mass and energy. If on the other hand the proton component is depleted by the reach of a rigidity dependent end of the injection spectrum, and if sources are uniformly distributed, hard injection spectra with low energy cutoff, together with local sources, could explain the data $[15,16]$.
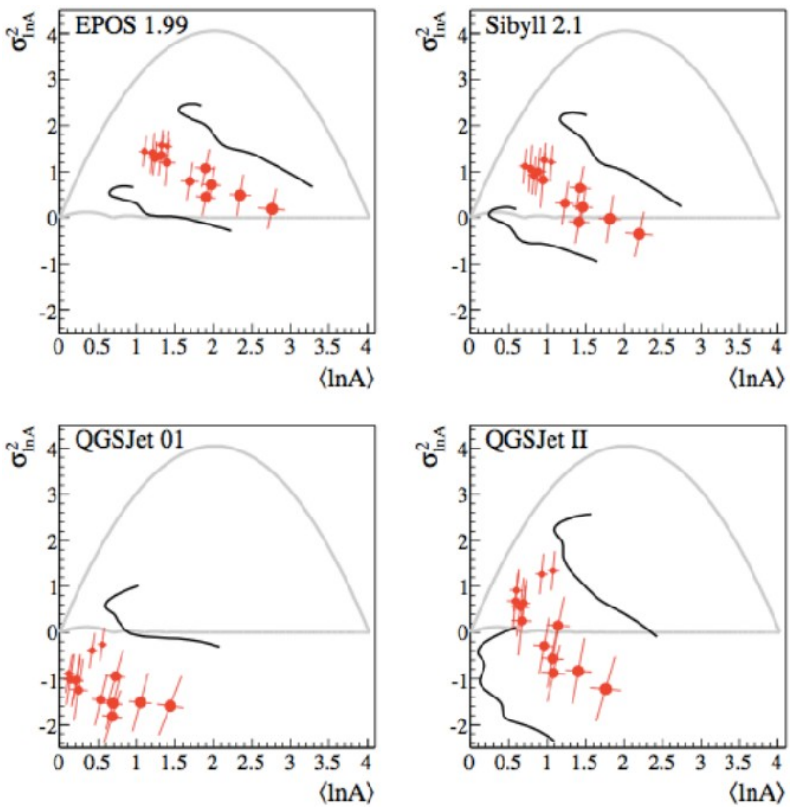

Figure 3: The Pierre Auger data in the $(<\ln A\rangle$ ,$\left.\sigma_{\ln A}^{2}\right)$ plane for different hadronic interaction models. Grey contours limit the allowed region, the systematic uncertainties are shown by the black lines.

\section{Primary Photons and Neutrinos}

UHE primary photons and neutrinos can provide invaluable information about the astrophysics of cosmic rays. Their detection would be a direct proof of the GZK cutoff; limits on exotic models [17] and tests for new physics [18] could be obtained from a positive or negative result on their detection. In both cases, their search is based on the characteristic features of the showers they produce in comparison to the hadronic ones.

Primary photons produce late developing showers, a characteristic further enhanced by the LPM effect [19]. The deeper $X_{\max }$, observable by the FD, is associated to a more dispersed distribution of the arrival time of the particles at ground level. At a given distance from the shower axis, the arrival time of the first particles is delayed with respect to a planar shower front and the radius of curvature is thus expected to decrease for photon induced showers. These observables can be recorded by means of the SD.

The upper limits derived from both the SD and the hybrid data collected by Auger are shown in Fig.4 and discussed in [20, 21]. Astrophysical scenarios are favoured with respect to top-down models.

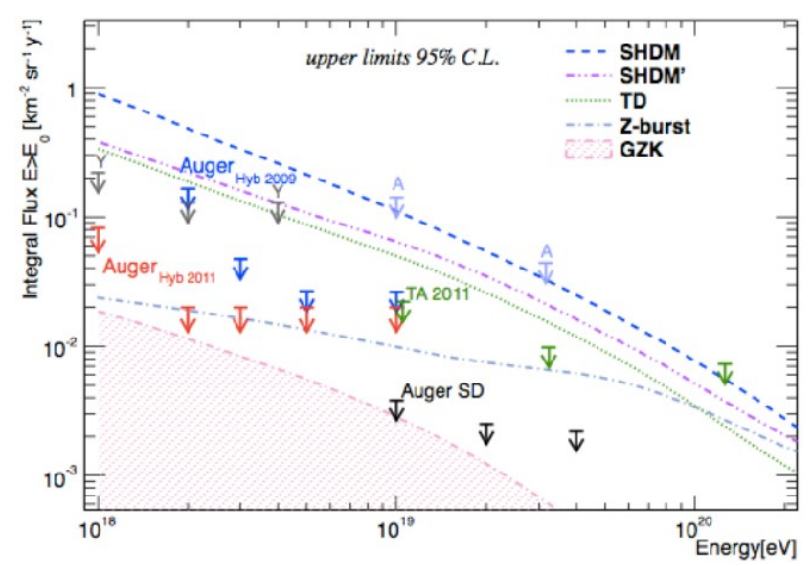

Figure 4: Upper limits on the integral photon flux. Different expectations are shown for comparison.

Primary neutrinos can produce showers characterized by a significant electromagnetic component; the huge hadronic background can be eliminated by looking at large zenith angles. $\nu_{\tau}$ can interact by charged current in the Earth crust, producing Earth-skimming upward-going showers; neutrinos of any flavour can interact in the atmosphere by neutral or charged current giving rise to downward-going events. Assuming a diffuse neutrino flux $\simeq k E^{-2}, 90 \%$ CL limits on their flux are obtained, as shown in Fig.5.

Neutrinos from point sources were also searched for, over a broad declination range (north of $\simeq-65^{\circ}$ and 
south of $\simeq 55^{\circ}$ ), for $E_{\nu}>10^{17} \mathrm{eV}$. For a differential neutrino flux $\simeq k_{P S} E^{-2}, 90 \%$ CL limits of $\simeq 5 \cdot 10^{-7}$ and $2.5 \cdot 10^{-6} \mathrm{GeV} \mathrm{cm} \mathrm{cm}^{-2} \mathrm{~s}^{-1}$ have been obtained for up-going and down-going events respectively [22].

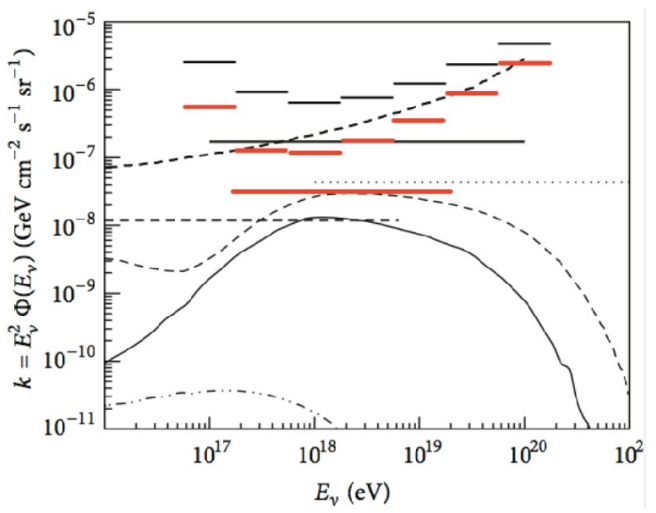

Figure 5: Differential and integrated upper limits on the single flavour $E^{-2} \nu$ flux (thin black lines: downward-going $\nu$, thick red line: Earth-skimming $\nu$ ). Different models are shown for comparison and discussed in [23]

\section{Anisotropies}

The spatial distribution of the arrival directions of UHE cosmic rays as a function of energy is a key observable to provide information about their sources and nature, complementary to those of energy spectrum and composition. Particles of UHE are most probably extragalactic, and if the observed cutoff in the spectrum can be attributed to the GZK propagation effect we could expect their sources to be confined in our courtyard, within about $100 \mathrm{Mpc}$.

In 2007 [24] the Auger Collaboration reported the observation of a correlation between the arrival directions of the highest energy cosmic rays and the positions of nearby AGN from the Véron-Cetty-Véron catalogue [25]. The result came from an analysis of indipendent data with a priori parameters determined from an exploratory scan; this allowed to avoid the use of penalty factors which would be needed in a posteriori analyses. The most recent update of this search is shown in Fig.6 [26]: the fraction of correlating cosmic rays is $(33 \pm 5) \%$ (28 events correlating out of a total of 84 ). The probability of this correlation to occur by chance if the true distribution of arrival directions is isotropic stays below $1 \%$. The independent averages of 10 consecutive events are also shown (black dots).

A recent comparison of our result with the Telescope Array and the Yakutsk ones showed that the correlating fractions are compatible [27]. More data are necessary to show whether this correlation is statistically significant or not.

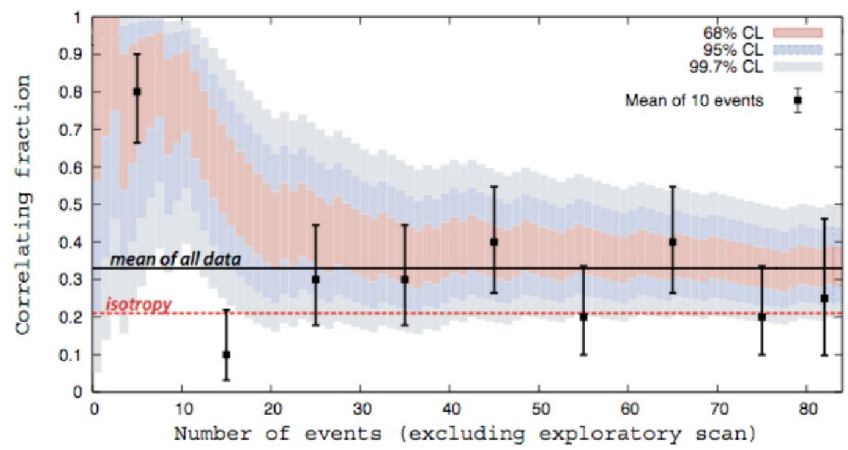

Figure 6: The correlating fraction as a function of the total number of time-ordered events. Different confidence levels are shown, together with the isotropy value $\mathrm{p}=0.21$ and the current estimate of all data, $p_{\text {data }}=0.33 \pm 0.05$.

Another possible scenario is that the anisotropy is dominated by cosmic rays originating from the vicinity of Centaurus A, the nearest active galaxy with an estimated distance of about $3.8 \mathrm{Mpc}$, since 19 events out of 7.6 expected have arrival directions within $24^{\circ}$ of CenA. A Kolmogorov-Smirnov test shows that the chance probability for this to happen is $4 \%$. Directionally aligned events, or "multiplets", can be expected from the same source after deflection in the magnetic fields, showing a correlation between the arrival direction and the inverse of energy. The largest multiplet found was one 12-plet, but also in this case the probability for it to come from an isotropic distribution is $\simeq 6 \%[28]$.

Potential sources of galactic cosmic rays have been looked for by performing a blind search for neutron primaries [29]. In fact, due to the relativistic time dilatation the UHE neutron mean decay length is $(9.2 \times$ $E / E e V$ ) kpc; above $2 \mathrm{EeV}$, neutron emitters can be searched for in the whole Galaxy. Auger can detect neutron showers by a simple search for an excess of proton-like showers from a specific direction in the sky. No candidates have been found, bringing to a median flux upper limit of $0.0114 \mathrm{n} \mathrm{km}^{-2} \mathrm{yr}^{-1}$ above $1 \mathrm{EeV}$. The absence of a neutron flux from the Galaxy, which could be expected in the hypothesis of sources steadily emitting protons and neutrons with similar luminosity, could be a hint that the sources at EeV energy could be e.g. extragalactic, transient or weak but densely distributed.

The large scale distribution of the arrival directions of cosmic rays is another fundamental tool in the search for their origin. The results from a study performed using data from the SD array are shown in Fig.7 [30]. No significant anisotropies are observed, resulting in the most stringent bounds on the first harmonic amplitude above $2.510^{17} \mathrm{eV}$. 

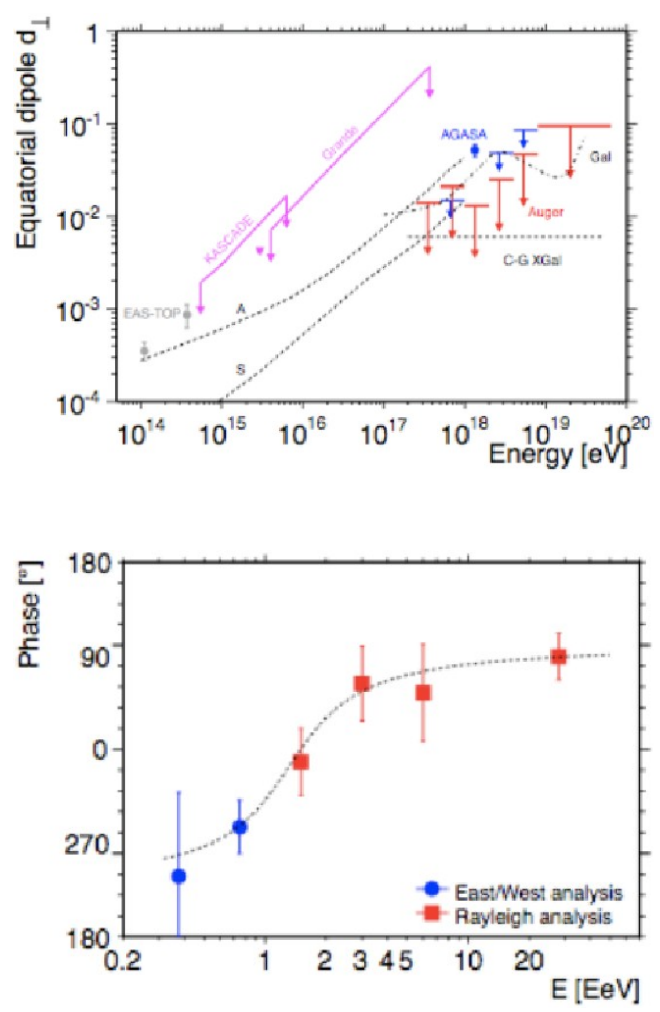

Figure 7: Equatorial dipole component (top) and phase of the first harmonic (bottom) as a function of energy.

The obtained limits already exclude some of the galactic models (labeled in Fig.7 with $A$ and $S$, indicating antisymmetric and symmetric galactic magnetic fields), according to which the cosmic rays at these energies are galactic and can escape by diffusion and drift motion. In the model labeled $\mathrm{Gal}$, cosmic rays are assumed to be galactic at all energies, and the anisotropy is due to purely diffusive motion caused by the turbulent component of the galacic magnetic field. In extragalactic models, the transition is put at the second knee and the cosmic rays large scale distribution is influenced by the relative motion of the observer with respect to the frame of the source. Assuming that the frame in which the cosmic ray distribution at these energies is isotropic is coincident with the cosmic microwave background rest frame, a small anisotropy (the extragalactic Compton-Getting effect, labeled $C$ - $G X g a l)$ is expected. Interestingly, the phase of the first harmonic shows a smooth transition between a common phase of $\simeq 270^{\circ}$ below $1 \mathrm{EeV}$ and $\simeq 100^{\circ}$ above $5 \mathrm{EeV}$. A consistency of the phase in ordered energy intervals can indeed be expected in presence of a real underlying anisotropy, standing out of the background more prominently than the amplitude. However, no confidence level can for the moment be assigned to this result, being an "a posteriori" observation.
The study of the large scale anisotropy has been performed for the first time with Auger data as a function of both the right ascension and the declination and expressed in terms of dipole and quadrupole amplitudes [31].

No significant deviations from isotropy are detected. Under the hypothesis that any anisotropy is dominated by these moments, the 99\% CL upper limits can be derived, as shown in Fig.8.
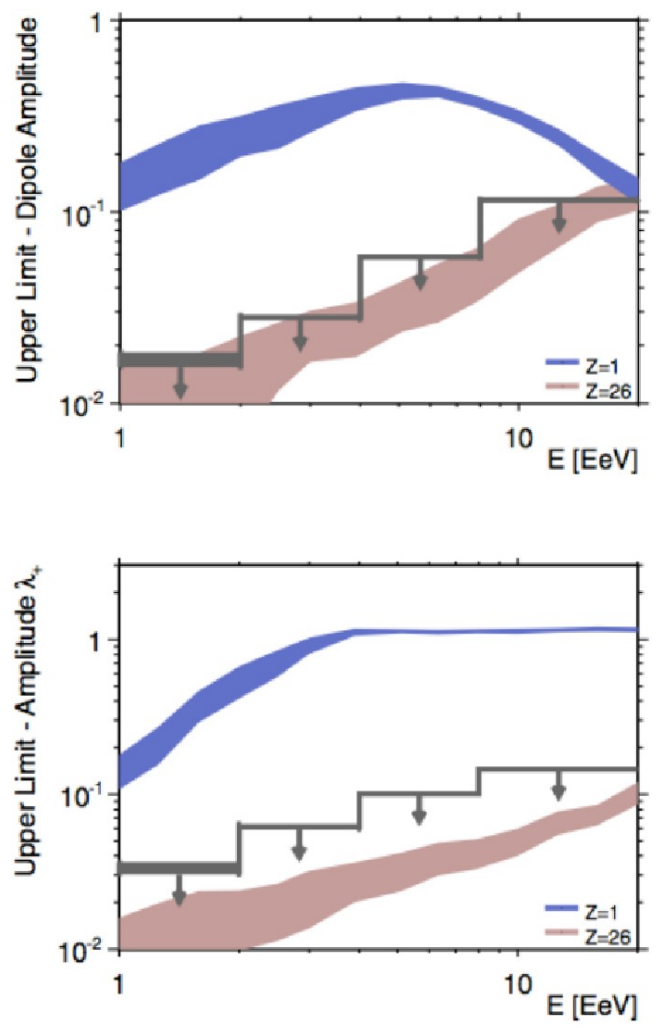

Figure 8: 99\% CL upper limits on the dipole and quadrupole momenta as a function of energy.

As an example of the power of the measurement to discriminate among different astrophysical models, the experimental limits are compared in the figure with the expectations from a toy model, in which the sources of protons and iron are stationary and uniformly distributed in the galactic disk. Being the expected amplitudes for protons largely above the allowed upper limits, we can exclude this scenario for the light component of $\mathrm{EeV}$ primary cosmic rays.

\section{$6 \quad$ Future Developments}

The Pierre Auger Observatory has reached a cumulative exposure of more than $26000 \mathrm{~km}^{2} \mathrm{sr}$ yr. New information about the characteristics of the primary cosmic rays have been derived, opening at the same time more questions and pointing to the need of an 
extension of the life time of Auger.

The measurement of composition of the primary particles from the $\mathrm{EeV}$ region up to the highest energies has emerged as the key for hitting the hottest scientific questions: a) understand the origin of flux suppression discussed in Sect.2, if due to the reach of the maximum energy at injection or to the GZK effect (a clear signature of which would be the observation of a flux of primary photons and neutrinos); b) perform composition driven anisotropy searches; c) determine the energy at which the transition from galactic to extragalactic sources of cosmic rays takes place.

\section{Acknowledgement}

The author wishes to thank the organizers for the warm hospitality in Mondello and the stimulating and interesting discussions during the Workshop.

\section{References}

[1] V. Berezinski, A. Gazizov, S. Grigorieva: 2006, Phys.Rev.D 74, 043005. doi:10.1103/PhysRevD. 74.043005

[2] A.M. Hillas: 2006, in Cosmology, Galaxy Formation and Astroparticle Physics on the pathway to the SKA Klöckner, H.-R., Jarvis, M., Rawlings, S. (eds.) April 10th-12th 2006, Oxford, United Kingdom.

[3] K. Greisen: 1966, Phys. Rev. Lett. 16, 748; G.T. Zatsepin and V.A. Kuzmin: 1966, Sov. Phys. JETP Lett. 4, 78.

[4] The Pierre Auger Collaboration: 2010, Nucl.Instr.Meth. A 620, 227. doi:10.1016/j.nima. 2010.04.023

[5] The Pierre Auger Collaboration: 2008, Phys.Rev.Lett. 101, 061101. doi:10.1103/PhysRevLett.101.061101

[6] The Pierre Auger Collaboration: 2010, Nucl.Instr.Methods A613, 29.

[7] R. Pesce for the Pierre Auger Collaboration: 2011, in 32nd Int.Cosmic Ray Conf., Beijing, China; arXiv:1107.4809.

[8] M. Settimo for the Pierre Auger Collaboration: 2012, Eur.Phys.J. Plus 127, 87.

[9] B. Dawson et al. for the Pierre Auger, Telescope Array and Yakutsk Collaborations: 2012, in Int.Symposium on future directions in UHECR physics, CERN.
[10] P. Facal for the Pierre Auger Collaboration: 2011, in 32nd Int.Cosmic Ray Conf., Beijing, China; arXiv:1107.4804.

[11] K.-H. Kampert, M. Unger: 2012, Astrop.Phys. 35, 660 .

[12] E. Barcikowski et al. for the Pierre Auger, Telescope Array and Yakutsk Collaborations: 2012, in Int.Symposium on future directions in UHECR physics, CERN.

[13] J. Matthews: 2005, Astrop.Phys. 22, 387.

[14] The Pierre Auger Collaboration: 2013, JCAP 02, 026.

[15] A.M. Taylor, M. Ahlers, F.A. Aharonian: 2011, Phys.RevD84, 105007. doi:10.1103/PhysRevD.84.105007

[16] D. Allard: 2012, Astrop.Phys. 39-40, 33. doi:10.1016/j . astropartphys . 2011 .10.011

[17] P. Battacharije and G. Sigl: 2000, Phys.Rep.327, 109.

[18] M. Galaverni and G. Sigl: 2008, Phys.Rev.Lett.100, 021102.

[19] L.D. Landau, I.Ya. Pomeranchuk: 1953, Dokl.Acad.Nauk.92, 553; A.B. Migdal: 1956, Phys.Rev.103, 1811.

[20] The Pierre Auger Collaboration: 2009, Astrop. Phys.31, 399; 2008, Astrop. Phys.29, 243.

[21] V. Scherini for the Pierre Auger Collaboration: 2012, in Int.Symposium on future directions in UHECR physics, CERN.

[22] The Pierre Auger Collaboration: 2012, ApJ.Lett. L4, 755 .

[23] The Pierre Auger Collaboration: 2013, Adv.in High Energy Phys.Res., 2013, 708680.

[24] The Pierre Auger Collaboration: 2007, Science 318,938 .

[25] M.P. Véron-Cetty, P. Véron: 2006, A\&A 445, 773.

[26] K.-H. Kampert for the Pierre Auger Collaboration: 2011, Highlight talk in 32nd Int. Cosmic Ray Conf., Beijing, China.

[27] O. Deligny et al. for the Pierre Auger, Telescope Array and Yakutsk Collaborations: 2012, in Int.Symposium on future directions in UHECR physics, CERN. 


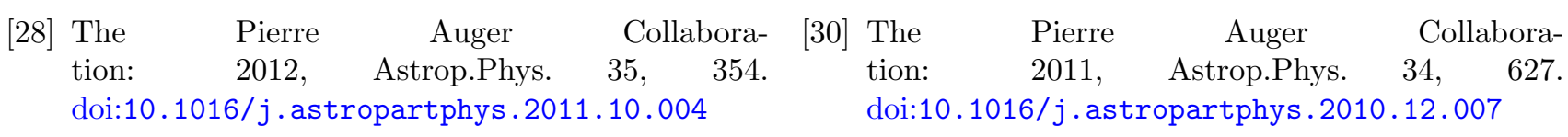

[29] The Pierre Auger Collaboration: 2012, ApJ 760, [31] The Pierre Auger Collaboration: 2012, ApJ.Suppl. 148. doi:10.1088/0004-637X/760/2/148 203, 34; 2013, ApJ.Lett. 762, L13. 